\title{
Three-dimensional computed tomography for evaluation of cervical spinal canal enlargement after en bloc open-door laminoplasty
}

\author{
Hisatoshi Baba, Kenzo Uchida, Yasuhisa Maezawa, Nobuaki Furusawa, Makoto Wada and Shinichi Imura \\ Department of Orthopaedic Surgery, Fukui Medical School, Shimoaizuki 23, Matsuoka, Fukui 910-11, Japan
}

\begin{abstract}
We evaluated the use of three-dimensional (spiral) computed tomography (CT) to assess widening of the bony cervical spinal canal following en bloc open-door C3-C7 laminoplasty in 31 patients. Measurements were performed using a computerised image processing system. The increment in the anteroposterior spinal canal axis and volume of the bony spinal canal was investigated in relation to changes in cervical spine lordosis as well as postoperative neurological improvement. Neurological follow-up averaged 2.6 years (range, 1 to 7 years). A mean increase of $42 \%$ was observed in the anteroposterior axis of the canal between $\mathrm{C} 3$ to $\mathrm{C} 7$ after surgery, while the volume increased by $45 \%$. Postoperatively, cervical spine lordosis correlated with increments in the volume of the enlarged bony canal rather than the anteroposterior axis. Postoperative neurological improvement correlated significantly with the increase in the canal volume. Our results indicate that spiral CT is useful for the assessment of the magnitude of cervical canal enlargement and evaluating the space available for the spinal cord following the relief of compression.
\end{abstract}

Keywords: three-dimensional computed tomography; cervical spine; laminoplasty; spinal canal; volume incrementation

\section{Introduction}

Three-dimensional (spiral) computed tomography (CT) scanning can be used to assess the surface and level of bone as well as joint structures. The exterior and splitsagittal views of the spiral CT show structural changes in the spine, which often require multiple twodimensional images to view the entire object. ${ }^{1-6}$

En bloc open-door laminoplasty for cervical myeloradiculopathy pertaining to multilevel compressive lesions, can widen the spinal canal at multiple segments, while simultaneously restructuring the posterior elements of the spine which are necessary for maintaining neck-extending musculature. ${ }^{7-11}$ The cervical cord can be well decompressed following surgery with possible dorsal migration of the cord within the enlarged canal in a stereotaxic fashion. ${ }^{12-14}$ The canal enlargement and posterior migration of the cerival cord are usually assessed by magnetic resonance imaging scanning. ${ }^{14-18}$ Serial CT scans may also be used to evaluate the degree of spinal canal enlargement at different vertebral levels, but such assessment often requires multiple transaxial images. A more useful approach would be to view the cervical spine and the enlarged canal and to identify those vertebrae associated with configurational changes in the spinal canal. To our knowledge, there are no reports that have

Correspondence: H Baba, MD, Associate Professor, Department of Orthopaedic Surgery, Fukui Medical School, Shimoaizuki 23, Matsuoka, Fukui 910-11, Japan previously assessed quantitatively the size of the cervical spinal canal after laminoplasty using spiral CT.

The present study was designed to assess spiral CT in evaluating the extent of cervical spinal canal enlargement and to quantify the configurational changes in the canal after open-door laminoplasty.

\section{Patients and methods}

\section{Patients and neurological assessment}

A total of 84 patients underwent en bloc open-door laminoplasty for multisegmental impingement on the cervical spinal cord during 1988-1995 in our unit. Among these, 31 patients (18 men and 13 women) consented to undergo serial spiral CT examinations before and after surgery. The protocol of this prospective study strictly followed the Ethical Committee Guidelines of our University.

The average age at surgery was 63.5 years (range, 40 to 81 years), and the average neurological as well as spiral CT follow-up was 2.6 years (range, 1 to 6.5 years). Nineteen patients had cervical spondylosis, while 12 patients had ossification of the posterior longitudinal ligament. Patients presented with a wide spectrum of clinical features of myeloradiculopathy. We used the Japanese Orthopaedic Association (JOA) scoring system to evaluate the neurological deficits in these patients (Table 1). ${ }^{19}$ 
En bloc open-door laminoplasty operation

Our surgical technique of en bloc open-door laminoplasty has been described previously in several other publications. ${ }^{7-9,14}$ Briefly, the patient was fixed with a three-point head rest, and a midline skin incision was made over the spinous process between $\mathrm{C} 2$ and $\mathrm{T} 1$ levels, followed by exposure of the laminae and bilateral facet joints from $\mathrm{C} 3$ to $\mathrm{C} 7$. Two longitudinal lateral troughs were made at sites just medial to the facet joints between $\mathrm{C} 3$ and $\mathrm{C} 7$. The inner cortices of the laminae on the neurologically advanced sides were excised with a high speed diamond burr and a microKerrison rongeur, whereas the contralateral hinged side of the laminae was thinly shaved. The laminae of $\mathrm{C} 3$ to C7 were carefully opened on the more affected side followed by spacer bone grafting between the facets and corresponding laminae usually at C4 and C6 levels, using the spinous processes harvested from $\mathrm{C} 6$ and $\mathrm{C}$,

Table 1 The Japanese Orthopaedic Association (JOA) scores for assessement of cervical myelopathy ${ }^{19}$

\begin{tabular}{cc}
\hline Categories & $\begin{array}{c}\text { Score } \\
\text { (points) }\end{array}$ \\
\hline
\end{tabular}

I. Motor function of the upper extremity

Impossible to eat with a spoon or chopsticks

Possible to eat with a spoon, but not with chopsticks

Possible to eat with chopsticks, but inadequate

Possible to eat with chopsticks, but awkward

Normal

II. Motor function of the lower extremity

Impossible to walk

Needs a cane or assistance on flat ground

Needs assistance only on stairs

Possible to walk without support, but slowly

Normal

III. Sensory function

(a) Upper extremity

Apparent sensory disturbance

Minimal sensory disturbance Normal

(b) Lower extremity

Apparent sensory disturbance

Minimal sensory disturbance Normal

0 point

1

2

3

(c) Trunk

Apparent sensory disturbance

Minimal sensory disturbance Normal

$I V$. Bladder function

Urinary retention or incontinence

Severe dysuria (sense of retention)

Slight dysuria (pollakiuria, retardation) Normal

1
2
3

0

1

0

1

0

1

2

1

2

3

A neurologically normal condition is the total of the best scores: $[\mathrm{I}+\mathrm{II}+\mathrm{III}+\mathrm{IV}]=17$ points or T1. A spacer bone graft, about 8 to $10 \mathrm{~mm}$ in length, was essential for the laminae so as to be maintained in the 'kept open' position postoperatively. However, the period for osseous union depends on the bone quality of the graft in individual patients. Isometric neck extension exercises were encouraged from the second week after surgery, and a soft neck collar fixation continued for approximately 3 months postoperatively. No patient in this series developed radiculopathy due to $\operatorname{root}(\mathrm{s})$ tethering or stretching following laminoplasty during the early postoperative period, and the patient was allowed to return to normal activity by $4-5$ months postoperatively.

\section{Radiological evaluation}

Lateral radiographs of the cervical spine, including neutral and flexion-extension views, were taken during the course of follow-up. Osseous union of the spacer bone graft was assessed primarily by serial CT scans. To obtain spiral CT images (Figure 1), we used a high resolution CT scanner (Highspeed Advantage RP, General Electric, Milwaukee, WI). The table incrementation speed was set at $3 \mathrm{~mm} / \mathrm{s}$, and a scan was

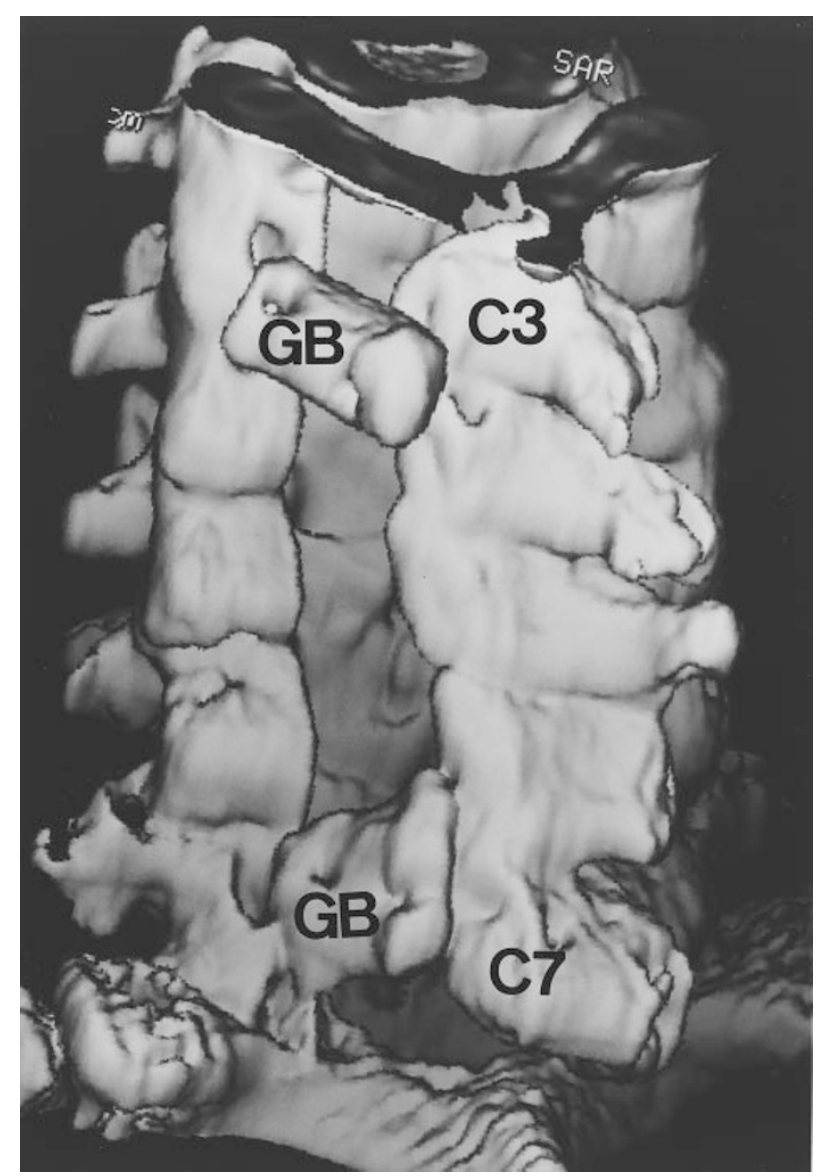

Figure 1 Exterior oblique view of the cervical spine after C3-C7 laminoplasty in a patient with multiple level spondylosis. C3: C3 lamina; C7: C7 lamina; GB: grafted bone 

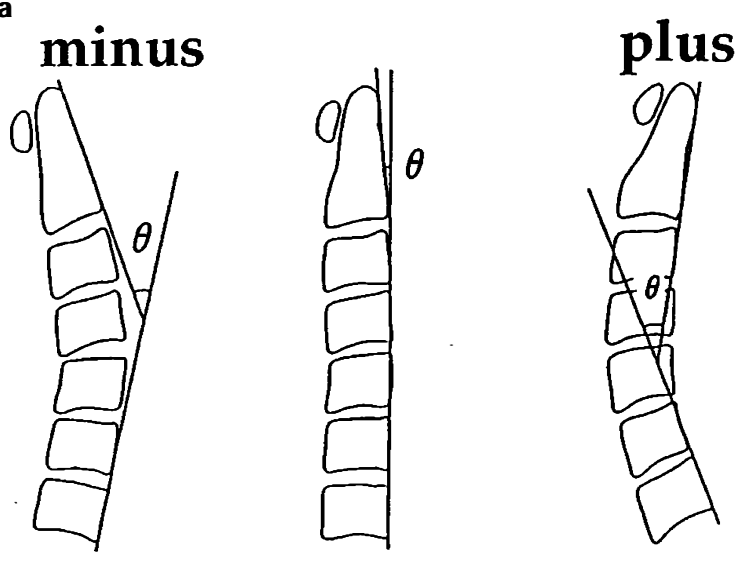

b
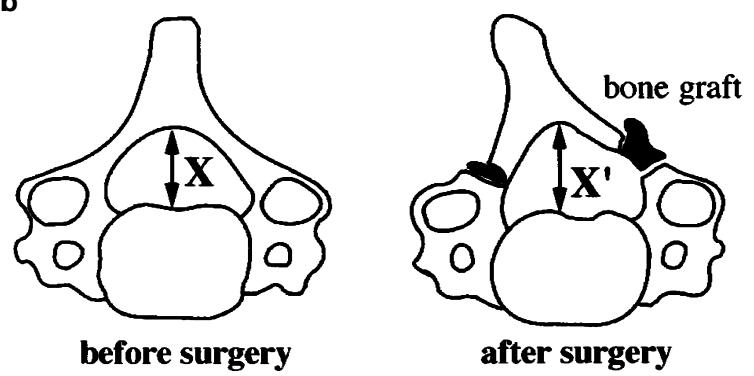

c

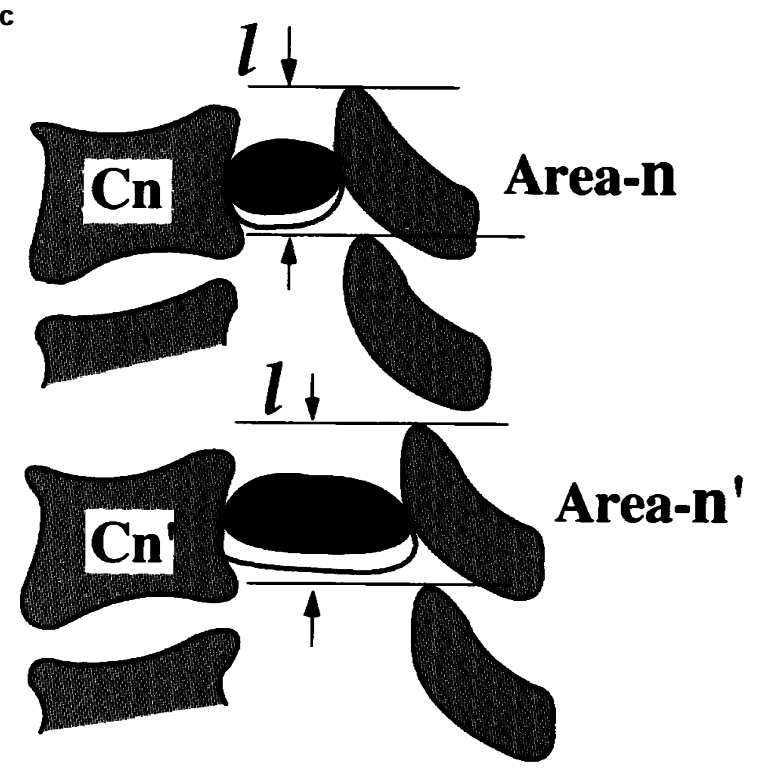

Figure 2 Schematic illustration of radiological measurements of the cervical spine. (a) Cervical spine lordosis angle is shown by the supplementary angle $(\theta)$. (b) The method used to measure changes in the anteroposterior dimension of the spinal canal. The anterior expansion ratio is determined by measuring $\mathrm{X}$ preoperatively and $\mathrm{X}^{\prime}$ postoperatively, as explained in the Methods. (c) The method used to measure changes in the volume of the cervical canal at each vertebra. Changes in the volume are determined by measuring the preand post-operative areas of the canal at a particular vertebra (area $n$, and area $n^{\prime}$, respectively; dark areas) and the length of the space $(l)$ extending between the anatomical landmarks, as described in the Methods obtained for every $3 \mathrm{~mm}$ slice. Reconstruction was performed every $1 \mathrm{~mm}$ pitch using $180^{\circ}$ interpolation. The databases scanned were displayed using an image analysis software (Advantage Window; General Electric, Milwaukee, WI), and the volume of the cervical canal was measured directly on spiral CT images. All images were stored on a Macintosh Quadra 650 computer (Apple, Cupertino, CA) for repeated analysis, using the NIH imaging software (Ohlandorf Research, Ottawa, ONT).

Radiological examination included (i) lordotic alignment of the cervical spine, designated as cervical spine lordosis angle, (ii) anteroposterior widening of the spinal canal, designated as spinal canal expansion ratio, measured by plain computed tomography, at each vertebral level, and (iii) increased volume of the cervical spinal canal at each vertebral level, designated as spinal canal volume increment ratio. Using the method we have previously reported, ${ }^{20}$ kyphosis or lordosis in the neutral film was defined by measuring the supplementary angle $(\theta)$ made by the two intersectioning lines drawn parallel to the posterior wall of the $\mathrm{C} 2$ and $\mathrm{C} 7$ vertebral bodies (Figure 2a). Negative values of angle $\theta$ represented kyphotic alignment of the cervical spine, while positive values indicated the presence of lordosis. Increased anteroposterior dimension of the spinal canal was evaluated using plain CT images taken before surgery and at follow-up, and represented the difference between $X^{\prime}$ and $X$ on Figure $2 b$. The spinal canal anteroposterior expansion ratio was then obtained by:

$$
\text { ratio }(\%)=\frac{X^{\prime}-X}{X} \times 100
$$

Volume measurements of the spinal canal were based on known cross-sectional areas of a series of contiguous slices within one vertebra and on the thickness of each slice. The area of the bony spinal canal at each vertebral level before laminoplasty and at follow-up, 'area- $n$ ' and 'area- $n$ ' ' in Figure 2c, was traced directly on the CT image displayed on the computer monitor and measured using the NIH imaging software. We also measured the distance ' $l$ ' between the upper ends of the laminae of two successive vertebrae (Figure 2c). The volume of the bony spinal canal at each vertebral level was represented by;

$$
\text { volume }\left(\mathrm{mm}^{3}\right)=\frac{\text { area } \times 1}{3}
$$

We also calculated the change in the volume of the canal at each vertebral level at follow-up. This was represented by:

Volume increment ratio $(\%)=$

postoperative volume - preoperative volume preoperative volume 
Statistical analysis

Data were expressed as mean \pm standard deviation. For statistical analysis, we used the Pearson's correlation analysis, one-way ANOVA, Wilcoxon signed rank test and the Fisher's post-hoc test, using the StatView II ${ }^{\circledR}$ program (Abacus Concepts, Berkely, CA). A probability of 0.05 was considered significant.

\section{Results}

Enlargement of the cervical spinal canal

Figure 3 shows the anteroposterior expansion and volumetric increment ratios of the spinal canal at $\mathrm{C} 3$ to C7. The spinal canal expansion ratio ranged from $37 \%$ at $\mathrm{C} 7$ to $45 \%$ at C6, with an average of $42 \pm 11 \%$. The widening was highest at $\mathrm{C} 6$ level compared with that at $\mathrm{C} 3$ and $\mathrm{C} 7$ levels $(P<0.05)$. On the other hand, the largest increase in the spinal canal volume occurred at $\mathrm{C} 4, \mathrm{C} 5$ and $\mathrm{C} 7$ levels, the ratio at these levels ranged from $42 \%$ to $49 \%$, with a mean ratio of $45 \pm 8 \%$ between $\mathrm{C} 3$ and $\mathrm{C} 7$ levels.

Correlation between cervical spine lordosis and widening of spinal canal

The relationships between postoperative cervical spine lordosis and spinal canal anteroposterior expansion ratio as well as volume increment ratio are shown in Figures $4 a$ and $b$, respectively. There was no significant correlation between postoperative cervical spine lordosis and spinal canal expansion ratio $\left(r^{2}=0.002\right.$, $P>0.05)$. In contrast, there was a significant correla-

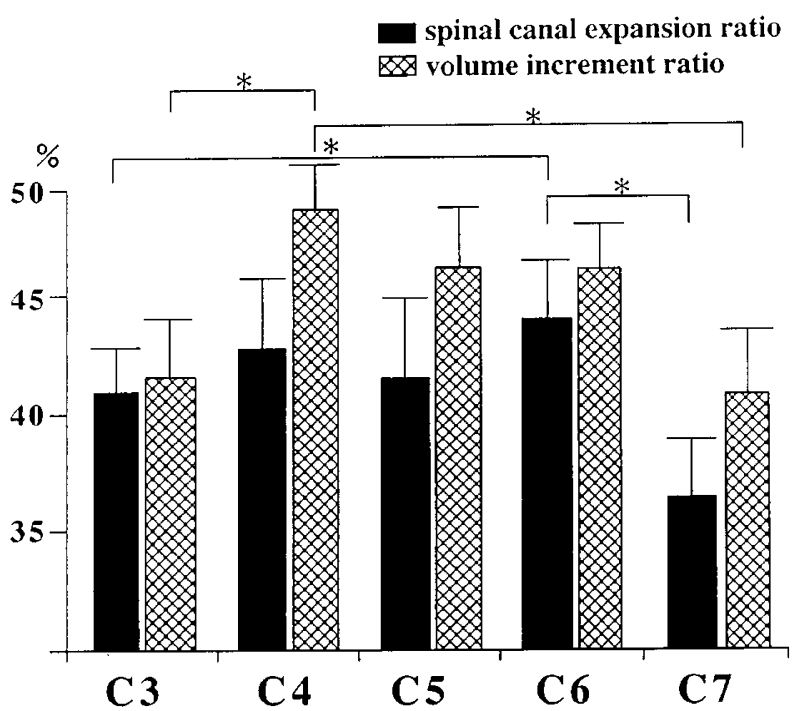

Figure 3 Spinal canal anteroposterior expansion ratio and volume increment ratio at each vertebral level after surgery. Data represent the mean \pm standard deviation of the entire group. ${ }^{*} P<0.05$ (one-way ANOVA and Fisher's post-hoc test)

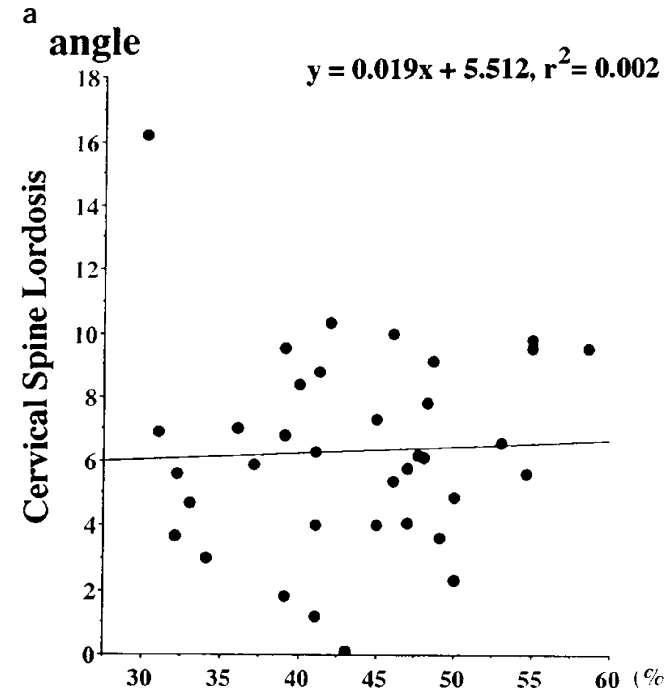

Spinal Canal Expansion Rate

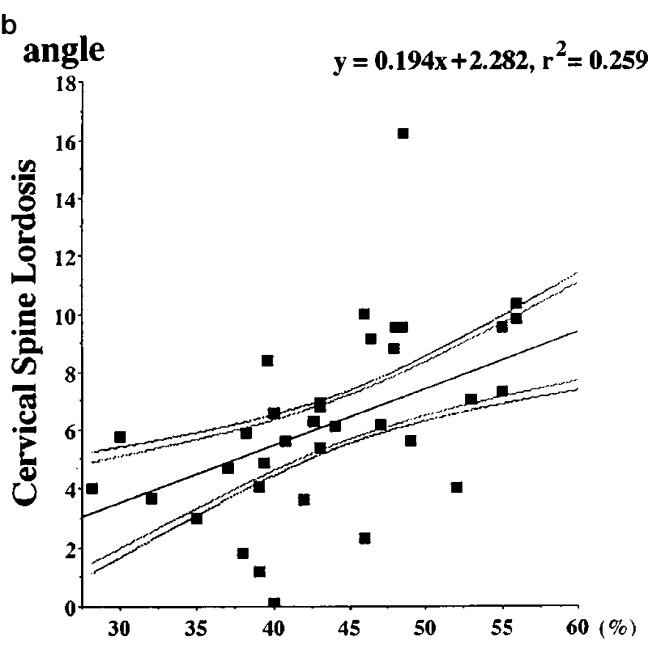

Volume Increment Ratio

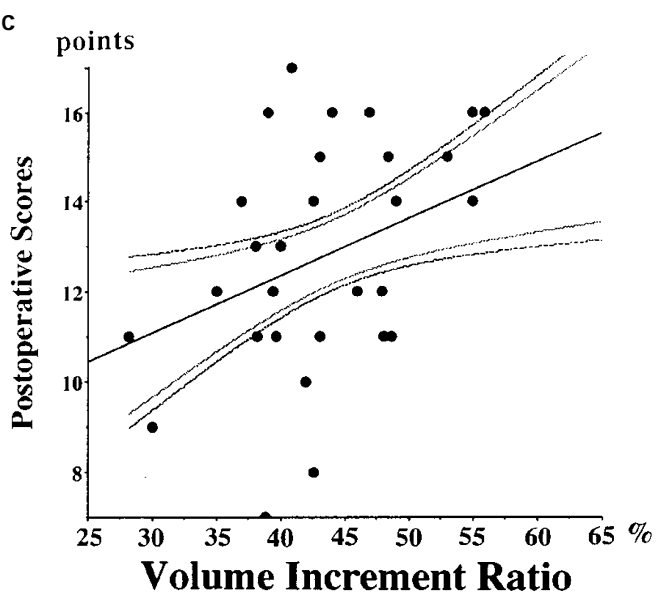

Figure 4 Relationship between the postoperative cervical spine lordosis ratio and spinal canal anteroposterior expansion ratio (a) and volume increment ratio (b). Significant correlation between postoperative Japanese Orthopaedic Association scores (abscissa) and volume increase of the spinal canal is shown (c) 
tion between the volume increment ratio and postoperative cervical spine lordosis ratio $\left(r^{2}=0.259\right.$, $P<0.05)$.

\section{Correlation between spinal canal enlargement and} postoperative Japanese Orthopaedic Association scores Surgery improved the JOA score from a preoperative mean of $9.96 \pm 3.35$ to $13.57 \pm 2.90$ points. The postoperative JOA scores correlated significantly with the increase in the volume of the spinal canal $(P<0.05$; Figure 4c).

\section{Discussion}

A spiral CT scan displays the surface of an object in a stereotaxic image. Such image provides valuable information for establishing the surgical plan and identifying the structural changes postoperatively. The laminoplasty operative technique allows the compromised cervical cord to migrate and extend posteriourly in close association with the enlargement of the bony spinal canal. $8,9,14,20-23$ Obviously, such increase in space would diminish the likelihood of compressive lesions of the spinal cord. Based on these considerations, a better evaluation of the size of the bony canal is beneficial in postoperative assessment and neurological prognosis.

Using the same operation technique in another group of patients, we previously reported a mean increase of $40 \%$ in the anteroposterior dimension of the bony canal. ${ }^{7,8,14}$ Other investigators also reported 30 to $70 \%$ increase in the canal size following laminoplasty. ${ }^{10}$ In the present study, the increase in the sagittal-plane diameter of the canal was similar to these early results and averaged $42 \%$ (range, 37 to $45 \%$ ). However, evaluation of the spiral CT showed a larger increase in the volume of the spinal canal compared with one-dimensional parameter of the anteroposterior expansion ratio.

The boomerang-shaped neural arch allows the compressed spinal cord to extend posteriorly and posterolaterally, when one side of the laminae is opened following laminoplasty. The spinal cord can move not only posteriorly but posterolaterally as well. Theoretically speaking, therefore, the volumetric effect of laminoplasty on the compromised cord is more accurately assessed by evaluating the volume increment ratio compared with the conventional onedimensional anteroposterior expansion rate measured on plain CTs. The present study showed significantly higher increases in the spinal canal volume at the level of $\mathrm{C} 4-\mathrm{C} 6$ vertebrae compared with those measured using one-dimensional scans.

Cervical laminoplasty is known to reduce lordosis of the spine although it ameliorates spinal cord decompression posteriorly. Strictly speaking, as Aboulker et $a l^{12}$ and Batzdorf and Batzdorff ${ }^{13}$ have suggested, the reduction in lordosis, or kyphosis, is inconvenient for the posterior translocation of the spinal cord so as to avoid anterior compression. In two recent studies from our group, insignificant reduction of lordosis was noted after laminoplasty. ${ }^{14,24}$ Although this reduction in the lordotic alignment of the postoperative cervical spine did not significantly affect neurological improvement, biomechanically, it may be inconvenient for the cord to be released from the lesions at posterior vertebral rims. By evaluating the spinal canal using a threedimensional approach, the present series showed that reductions of lordosis were associated with reduced volume of the cervical spinal canal postoperatively. In contrast, no such relationship was present with anteroposterior spinal canal expansion ratio. These findings indicate that the use of spiral CT is useful particularly for postoperative evaluation of those patients who present with considerable decrease of lordosis postoperatively associated with unfavourable neurological recovery.

At present time, spiral CT and measurement of spinal canal volume are, unfortunately, time-consuming and rather difficult to perform during routine clinical practice. In addition, the patient might be exposed to a higher dose of irradiation during imaging. However, these difficulties may resolve with further advancement in radiological technology and software development. Due to these limitations, we recommend the use of spiral CT imaging only in the presence of complex structural deformities of the cervical spine, graft dislodgment or when the weak laminae is liable to failure. However, when the resources are not available to obtain a spiral CT, it should be kept in mind that after the laminoplasty operation, a considerable spatial increase in the volume of the cervical canal has occurred, more markedly than measured on the serial CT images.

Based on the results of the present study, we suggest that spiral CT imaging is useful for assessing the increase in the volume of the cervical spinal canal following laminoplasty. The enlarged spinal canal can be more correctly evaluated with this method than conventional serial $\mathrm{CT}$ imaging. Postoperative reduction of lordotic alignment significantly affects volumetric increment of the enlarged spinal canal.

\section{Acknowledgements}

This work was supported by grants from the Japanese Orthopaedics and Traumatology Foundation Incorporated (grant no. 0082: Maruho Award), and the Public Health Bureau of the Japanese Ministry of Public Health and Welfare. We thank Professor FG Issa, University of Sydney, Sydney, Australia, for kindly reviewing the manuscript.

\section{References}

1 Jurik AG, Jensen LC, Hansen J. Radiation dose by spiral CT and conventional tomography of the sternoclavicular joints and the manubrium sterni. Skeletal Radiol 1996; 25: 467-470. 
2 Kalender WA, Seissler W, Klotz E, Vock P. Spiral volumetric CT with single-breath-hold technique, continuous transport, and continuous scanner rotation. Radiology 1990; 176: $181-183$.

3 Kuszyk BS, Heath DG, Bliss DF, Fishman EK. Skeletal 3-D CT: advantages of volume rendering over surface rendering. Skeletal Radiol 1996; 25: 207-214.

4 Scapinelli R. Three-dimensional computed tomography in infantile atlantoaxial rotatory fixation. J Bone Joint Surg [Br] 1994; 76: $367-370$

5 Smith GA, Aspden RM, Porter RW. Measurement of vertebral foraminal stenosis using three-dimensional computed tomography. Spine 1993; 18: 629-636.

6 Zinreich SJ. Three-dimensional computed tomography of the spine. In: Roland LR (ed). Spine: State of the Art Review; Spinal Imaging. Hanley \& Belfus: Philadelphia 1995, pp 287-297.

7 Baba H, Furusawa N, Chen Q, Imura S. Cervical laminoplasty in patients with ossification of the posterior longitudinal ligaments. Paraplegia 1995; 33: $25-29$.

8 Baba $\mathrm{H}$ et al. Osteoplastic laminoplasty for cervical myeloradiculopathy secondary to ossification of the posterior longitudinal ligament. Int Orthop 1995; 19: 40-45.

9 Baba $\mathrm{H}$ et al. Laminoplasty with foraminotomy for coexisting cervical myelopathy and unilateral radiculopathy: a preliminary report. Spine 1996; 21: $196-202$

10 Kawai S. Cervical laminoplasty. In: Bridwell KH, DeWald RH (eds) The Textbook of Spinal Surgery, Vol. 2. JB Lippincott: Philadelphia 1991; pp 805-812.

11 Skowronski J, Bielecki M. The results of laminectomy and laminoplasty in cervical myeloradiculopathy. Rocz Akad Med Bialymst 1992; 37: 71 - 73 .

12 Aboulker $\mathbf{J}$ et al. Les myélopathies cervicales d'origine rachidienne. Neurochirurgie 1965; 11: 87 - 198.

13 Batzdorf U, Batzdorff A. Analysis of cervical spine curvature in patients with cervical spondylosis. Neurosurgery 1988; 22: 827 836.
14 Baba $\mathrm{H}$ et al. Lordotic alignment and posterior migration of the spinal cord following en bloc open-door laminoplasty for cervical myelopathy: a magnetic resonance imaging study. J Neurol 1996; 243: $626-632$.

15 Batzdorf U, Flannigan BD. Surgical decompressive procedures for cervical spondylotic myelopathy: a study using magnetic resonance imaging. Spine 1991; 16: $123-127$.

16 Levi L et al. The significance of dorsal migration of the cord after extensive cervical laminectomy for patients with traumatic central cord syndrome. J Spinal Disord 1995; 8: 289-295.

17 Okada Y et al. Magnetic resonance imaging study on the results of surgery for cervical compression myelopathy. Spine 1993; 18: $2024-2029$.

18 Yone K, Sakou T, Yanase M, Ijiri K. Preoperative and postoperative magnetic resonance image evaluations of the spinal cord in cervical myelopathy. Spine 1992; 17: S388 - S392.

19 Japanese Orthopaedic Association. Criteria on the evaluation of treatment of cervical myelopathy. J Jpn Orthop Assoc (Tokyo) 1976; 49: addendum no. 5.

20 Baba $\mathrm{H}$ et al. Late radiographic findings after anterior cervical fusion for spondylotic myeloradiculopathy. Spine 1993; 18: $2167-2173$.

21 Transfeldt EE. Cervical spondylosis. In: Bridwell KH, DeWald RL (eds) The Textbook of Spinal Surgery. JB Lippincott: Philadelphia 1991, pp 771-804.

22 Herkowitz HN. A comparison of anterior cervical fusion, cervical laminectomy, and cervical laminaplasty for the surgical management of multiple level spondylotic radiculopathy. Spine 1988; 13: $774-780$.

23 Frank E, Keenen TL. A technique for cervical laminoplasty using mini plates. Br J Neurosurg 1994; 8: 197-199.

$24 \mathrm{Baba} \mathrm{H}$ et al. Flexibility and alignment of the cervical spine after laminoplasty for spondylotic myelopathy: a radiographic study. Int Orthop 1995; 19: $116-121$. 